\title{
An outbreak of severe respiratory tract infection caused by human metapneumovirus in a residential care facility for elderly in Utrecht, the Netherlands, January to March 2010
}

M J te Wierik (Mtewierik@ggdmn.nl) ${ }^{1}$, D T Nguyen², M F Beersma², S F Thijsen³, K A Heemstra3,4

1. Municipal Health Service Midden-Nederland, Zeist, the Netherlands

2. Department of Virology, Erasmus Medical Centre, Rotterdam, the Netherlands

3. Department of Medical microbiology, Diakonessenhuis, Utrecht, the Netherlands

4. Department of Medical microbiology, University Medical Centre Utrecht, Utrecht, the Netherlands

Citation style for this article:

te Wierik MJ, Nguyen DT, Beersma MF, Thijsen SF, Heemstra KA. An outbreak of severe respiratory tract infection caused by human metapneumovirus in a residential care facility for elderly in Utrecht, the Netherlands, January to March 2010.

Euro Surveill. 2012;17(13):pii=20132. Available online: http://www.eurosurveillance.org/ViewArticle.aspx?Articleld=20132

Article published on 29 March 2012

Recognition of infections with human metapneumovirus (HMPV) among institutionalised elderly is rising. When HMPV was found to be the causative agent of an outbreak of pneumonia in a residential care facility for elderly in the Netherlands, an elaborate outbreak investigation was set up, including active surveillance for new cases. From clinical cases, defined by fever $(>$ $38^{\circ} \mathrm{C}$ ) and symptoms of respiratory tract infections, respiratory samples for analyses of viral pathogens by real-time Reverse Transcriptase Polymerase Chain Reaction (rRT-PCR) and blood samples for determination of HMPV-specific IgM and IgG antibody titres were taken. Five staff members and 18 residents fulfilled the clinical case definition. Of those, five residents tested positive for HMPV by rRT-PCR. The combination of rRTPCR and serology identified nine confirmed cases, six probable cases, six possible cases and ruled out two persons as cases. Among residents, the outbreak of HMPV had an attack rate, ranging from $5 \%$ for laboratory-confirmed cases, to $13 \%$ for clinical cases. This outbreak investigation shows that HMPV is a potential serious pathogen for institutionalised elderly.

\section{Introduction}

Outbreak description

In mid-February 2010, staff of a residential care facility for the elderly notified an outbreak of pneumonia to the municipal health service of the town of Utrecht, the Netherlands. At the time, in five weeks, nine of the 140 residents and two staff members had fallen ill with pneumonia. Three residents had been admitted to the hospital. One resident was admitted with cardiac failure and pneumonia, another with dyspnoea (oxygen dependent) due to a lower respiratory tract infection (RTI) (X-ray: possible lobar infiltrate) and the third resident was admitted among other things because of a deteriorating lower RTI (X-ray: lobar infiltrate). All three died in the hospital shortly after admission. No virological examination had been performed and a definite causative agent was not established for any of the three deceased patients. The other ill residents were not admitted to hospital but treated by general practitioners in the care facility, mostly with antibiotics. Although RTIs can be expected in winter season, the clinical presentation - especially the three deaths with a possible common causative agent - together with the number of cases, were found severe enough to justify an outbreak investigation.

This induced an inventory of clinical cases (onset of disease, symptoms, clinical diagnosis, treatment, date of birth and sex) and the active surveillance for new clinical cases among residents and staff members in this facility, from mid-February onwards. A clinical case was defined by fever $\left(>38^{\circ} \mathrm{C}\right)$ and clinical symptoms of RTI. Respiratory samples for analyses of viral pathogens were taken from three new clinical cases, five days after the initial notification. When laboratory investigations on 23 February 2010 identified human metapneumovirus (HMPV) as causative agent, an elaborate outbreak investigation among staff and residents was set up.

Residents, staff members and the general practitioners of the residents were informed about the outbreak and infection control measures were taken. These included: (i) isolation of the ill residents in their own apartments until symptoms resolved, (ii) use of a surgical mask by staff members while giving care to the ill residents as well as application of strict hand hygiene, (iii) all persons - staff members, family members, and other visitors - leaving the apartment of ill residents were advised to apply hand alcohol. 
These measures were lifted on 15 March 2010 after two maximum incubation periods (12 days) without the occurrence of new clinical cases.

\section{Background on human metapneumovirus}

HMPV is a respiratory pathogen, which was first identified in 2001 in children with RTI [1]. It is a singlestranded RNA virus with a lipid envelope and belongs to the family Paramyxoviridae, subfamily Pneumovirinae. Phylogenetic analysis has identified two subgroups of HMPV, subgroups $A$ and $B$, and two clades within each of these subgroups [2]. Seroprevalence data suggest that most children are infected by the age of five and re-infection occurs throughout life $[1,3,4]$. Most infections occur during late winter and early spring [4]. Depending on the region of the world, both HMPV subgroups $A$ and $B$ may co-circulate, but during an epidemic one subtype usually dominates [5]. Transmission is likely by direct (e.g. via hands) or close contact (e.g. via coughing or sneezing) with contaminated secretions, which may involve large particle aerosols, droplets or saliva. The clinical incubation period is not precisely known; estimates range from three to six days [6]. Clinical manifestations of HMPV infection are similar to those of respiratory syncytial virus (RSV). The majority of HMPV infections are self-limiting mild upper RTI, but in a minority it causes lower RTI such as bronchiolitis and pneumonia, requiring occasionally mechanical ventilation support. Risk factors for severe HMPV infections are age ( $<5$ years and $>65$ years of age), compromised immune status and underlying pulmonary or cardiac disease $[7,8]$. Treatment is supportive and varies with the clinical manifestations. Ribavirin and polyclonal intravenous immune globulin (IVIG) are active against HMPV in vitro and reduce viral replication in experimentally infected mice, but clinical data on the effectiveness in humans are lacking $[9,10]$. Since the discovery of HMPV the majority of clinical publications concerns infections in children, but the number of publications on outbreaks of HMPV infections in institutionalised adults and elderly is rising [11-15]. However, no studies have been performed combining results of real-time Reverse Transcriptase Polymerase Chain Reaction (rRT-PCR) and serology during outbreaks.

This article describes an outbreak of HMPV in a residential care facility for elderly, in which a combined approach of epidemiology and laboratory investigations (rRT-PCR and serology) gave insight in the extent of HMPV infection.

\section{Methods}

\section{Laboratory investigation}

Respiratory samples for viral analysis were taken from each new clinical case. Sampling consisted of a nasal swab and a pharyngeal swab which were transported to the laboratory in one viral transport medium (universal transport medium (UTM)). Viral analysis was performed with rRT-PCR.
Sampling new clinical cases for viral analysis continued until the outbreak was ended on 15 March 2010. Furthermore, cases positive for HMPV were periodically sampled until the last respiratory sample tested negative for HMPV, with a sampling interval of approximately eight days.

The initial viral analysis by rRT-PCR was performed by the laboratory of one of the local hospitals. In a later stage, all respiratory samples were analysed in one batch by the Erasmus Medical Centre, Rotterdam, which is one of the two national reference laboratories for respiratory viruses.

After viral DNA or RNA isolation from nasopharyngeal swab supernatant, a multiplex rRT-PCR respiratory virus panel was used to identify viruses (HMPV, RSV types $A$ and $B$, rhinovirus, parainfluenza virus 1-3, adenovirus and influenza $A$ and $B$ viruses) as previously described [16-19].

To culture HMPV, it was propagated as described previously [8]. LLC-MK2 cells (monkey kidney cells, ATCC CCL-7) at 80-90\% confluency were inoculated in culture medium supplemented with trypsin. Subsequently, each following day, cells were monitored for cytopathogenic effect and harvested when maximum cytopathic effect was observed. All isolations were performed in duplicates. Viral RNA was extracted, amplified by RT-PCR, sequenced and run on an ABI genetic analyser as described before with slight modifications [20].

To gain insight into the magnitude of the outbreak, all clinical cases - i.e. all clinical cases before and after outbreak notification - were requested to donate a blood sample for determination of HMPV-specific immunoglobulin (Ig) M and IgG antibody titres. A second sample was requested from the symptomatic staff members. Residents were requested to provide a second sample only if a HMPV infection was not probable or proven by the results of rRT-PCR or serology from the first sample.

HMPV-specific IgM and IgG antibody titres were determined using the direct immunofluorescent-antibody (IFA) test as described before [4,5]. Data are expressed as reciprocal anti-HMPV IgM and IgG antibody titres. All serological analyses were performed in duplicates. An anti-HMPV IgM antibody titre of 64 or higher was considered proof of HMPV infection. The same holds for a fourfold increase in anti-HMPV IgG antibody titres between the first and second blood sample taken (seroconversion). An isolated HMPV IgG titre of 256 or above was considered indicative of a recent HMPV infection.

\section{Final case classification}

Results of both analyses were combined into a final case classification for clinical cases: no proof of HMPV infection (serology and rRT-PCR negative), possible (insufficient laboratory investigations), probable (IgG titre first blood sample 256 or above), and confirmed 
HMPV infection (rRT-PCR positive or IgM first blood sample positive (IgM titre 64 or above) or seroconversion (fourfold increase in anti-HMPV IgG antibody titres between the first and second blood sample taken).

\section{Results}

Outbreak

In all, 23 persons were included in the outbreak investigation: five staff members (one male, four female) and 18 residents (five male, 13 female). Mean \pm Standard Deviation (SD) (minimum-maximum) age for staff members and residents, was $47.6 \pm 3.6(37-59)$ and $90.1 \pm$ $1.1(83-98)$ years respectively. Underlying conditions of the cases are shown in Table 1.

\section{TABLE 1}

Underlying conditions in clinical cases, outbreak of human metapneumovirus in a residential care facility for elderly in Utrecht, the Netherlands, January-March 2010 $(n=23)$

\begin{tabular}{|l|c|}
\hline Underlying condition & Number of cases \\
\hline Cardiac disease $^{\mathrm{a}}$ & 14 \\
\hline Cardiac failure $^{\mathrm{a}}$ & 6 \\
\hline Atrial fibrillation $^{\mathrm{a}}$ & 3 \\
\hline Angina pectoris $^{\mathrm{a}}$ & 2 \\
\hline Chronic obstructive pulmonary disease $^{\mathrm{a}}$ & 3 \\
\hline Hypertension & 4 \\
\hline Cerebro vascular attack $^{\mathrm{a}}$ & 5 \\
\hline \multicolumn{1}{|c|}{ Transient ischemic attack $^{\mathrm{a}}$} & 3 \\
\hline Diabetes mellitus $^{\mathrm{a}}$ & 4 \\
\hline Renal failure $^{\mathrm{a}}$ & 1 \\
\hline Hypothyroidism $^{\mathrm{a}}$ & 1 \\
\hline Asthma $^{\mathrm{b}}$ & 2 \\
\hline
\end{tabular}

a Underlying condition only in residents of the care facility for elderly.

b Underlying condition only in staff of the care facility for elderly.
Among the residents, there was no clustering of cases in wards or floors as they were scattered over 10 of the 12 floors of the residential care facility. Staff members were not only nursing staff. Twelve persons had an onset of disease after 17 February 2010 and therefore respiratory samples were taken. Among the new clinical cases, another two residents were hospitalised; one because of the seriousness of the RTI, the other because of another medical condition. Both were discharged in an improved condition after about 12 days. However, one patient died eventually.

Figure 1 shows the epidemic curve of the outbreak, as well as the timeline of outbreak management.

\section{Real-time Reverse Transcriptase}

Polymerase Chain Reaction results

Five of 12 respiratory samples, which were all from residents, tested positive for HMPV by rRT-PCR. Follow-up by rRT-PCR was possible for four residents: nose and throat swabs were obtained with an interval of approximately eight days until a sample tested negative. As shown in Figure 2, these residents shedded HMPV for at least nine to 17 days after onset of disease.

rRT-PCR results of both laboratories were consistent (Pearson's $r$ between the cycle threshold values (Ct-values) is $0.94, \mathrm{p}<0.001$, nine samples). There was a clear correlation between the Ct-values and the time from disease onset: the longer the period between onset of disease and respiratory sampling, the higher the Ct-value (Figure 3, $\mathrm{R}^{2}$ linear $=0.39$, p<0.04, $11 \mathrm{sam}$ ples), indicating lower virus levels with time.

The virus was isolated from one respiratory sample from HMPV positive patient 3 (Figure 2). HMPV genotype A was assigned based on submission of the glycoprotein (G) gene sequence to a BlastN search (Genbank accession JN200816).

\section{FIGURE 1}

Epidemic curve and outbreak management, outbreak of human metapneumovirus in a residential care facility for elderly in Utrecht, the Netherlands, January-March 2010

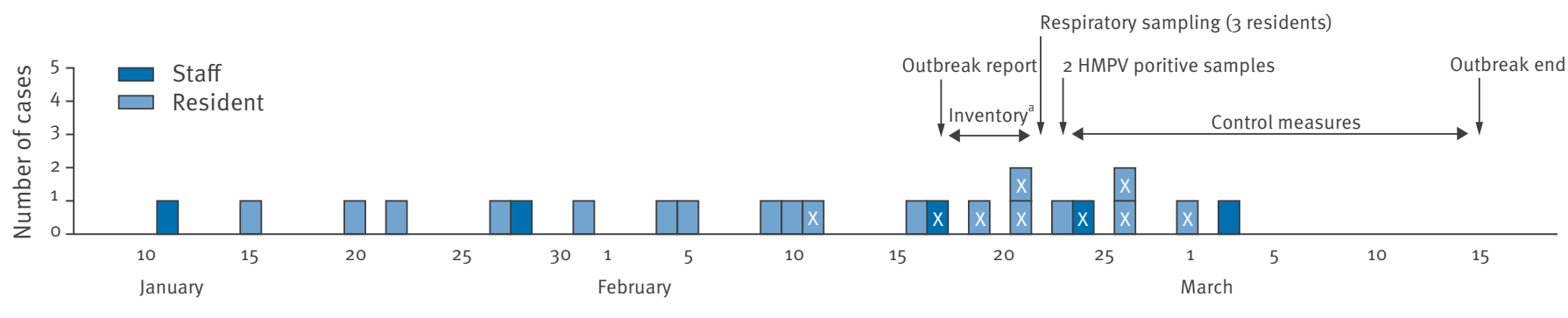

Date of symptom onset, 2010 


\section{Serological results}

The first serum sample was taken at a median of 23 days after onset of disease (range: $11-63, n=18)$. The second sample was taken at a median of 64 days after onset of disease (range: $59-113, n=9$ ). As at that time, the HMPV genotype was unknown, both HMPV A and HMPV B infected LLC-MK2 cells were used to determine the antibody titres. As sequencing showed it was HMPV genotype A, only HMPV A-specific antibody titres are shown (Table 2). The titres of HMPV genotype $B$ were similar or one step deviant from the titres of HMPV genotype A.

One of 18 clinical cases showed a positive HMPVspecific IgM antibody titre in the first serum sample; this case still had a positive IgM in the second sample. Anti-HMPV IgG antibody titres in both first and second

\section{FIGURE 2}

Follow-up of four human metapneumovirus positive cases, outbreak of human metapneumovirus in a residential care facility for elderly in Utrecht, the Netherlands, JanuaryMarch 2010

Onset of disease

$\square$ Case's sample testing positive for HMPV

Case's sample testing negative for HMPV

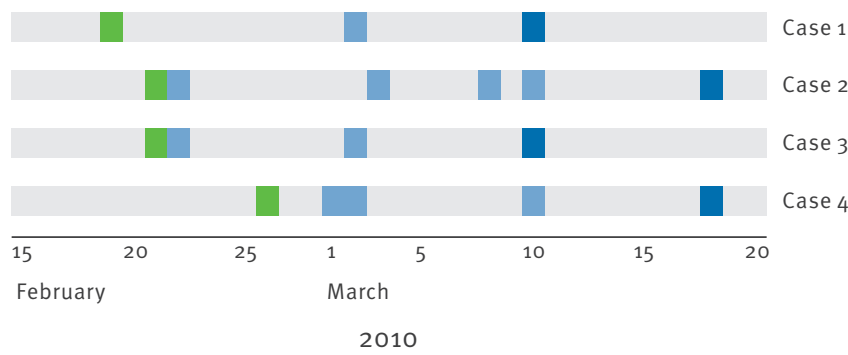

HMPV: Human metapneumovirus.

\section{FIGURE 3}

Correlation between real-time Reverse Transcriptase Polymerase Chain Reaction for human metapneumovirus Cycle threshold-values and number of days after disease onset

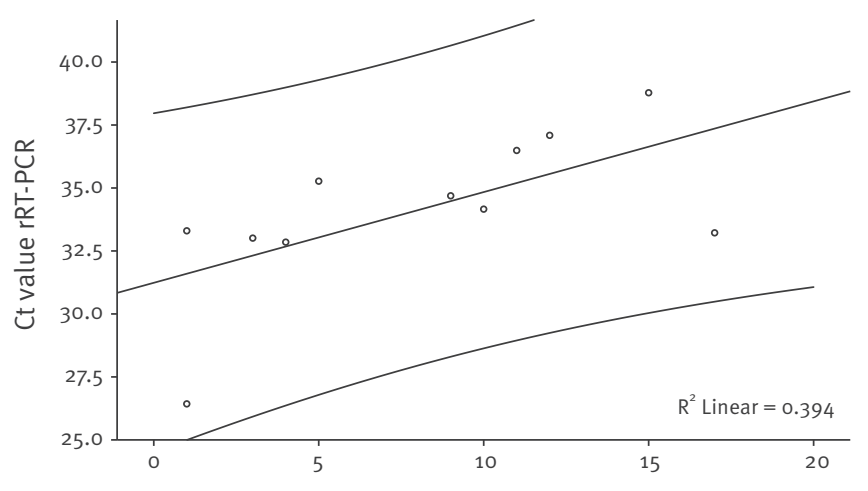

Number of days after onset of disease

Ct: Cycle threshold; rRT-PCR: real-time Reverse Transcriptase Polymerase Chain Reaction. sample were indicative of recent HMPV infection for six clinical cases. Seroconversion occurred in seven sera of nine persons tested twice (Table 2).

\section{Final case classification}

Table 2 shows the rRT-PCR and serological results as well as the final case classification of the 23 clinical cases. As the rRT-PCR results already identified five confirmed cases, the serological results increased that number to nine. Based on IgG level in the first serum sample, another six clinical cases could be classified as probable cases. The majority of the confirmed (seven of nine cases) and all probable cases (six cases) were residents. Nevertheless, the diagnostic approach identified two staff members as confirmed cases as well. For two clinical cases, both diagnostic results excluded a HMPV infection. Figure 4 summarises the results of the outbreak investigation.

Figure 4. Flowchart of the laboratory investigations and results, outbreak of human metapneumovirus in a residential care facility for the elderly, Utrecht, the Netherlands, January-March 2010

Given the numbers of possible, probable and confirmed cases, the attack rate of HMPV infection among residents in this outbreak was $5 \%$ for laboratory-confirmed cases (seven confirmed cases of 140 residents) and $13 \%$ for clinical cases (18 possible, probable and confirmed cases of 140 residents).

\section{Discussion}

This article describes an outbreak of HMPV in a residential care facility for elderly. Notifications of outbreaks of pneumonia in these kind of facilities for elderly are not very common in the Netherlands and HMPV as causative agent has not been described earlier.

Five of the 12 (42\%) clinical cases, occurring after the outbreak was notified and from whom respiratory samples were taken, tested positive for HMPV by rRT-PCR. Our results are in agreement with those of Boivin et al., who, upon investigating a HMPV outbreak in a long term care facility in Canada, found six of 13 tested residents (46\%) HMPV positive by rRT-PCR [11]. In a summer outbreak in a long term care facility in California, however, a lower proportion was found, with five of 20 cases (25\%) testing HMPV positive by RT-PCR [13]. Higher proportions than in our study are nevertheless also reported concerning two other outbreaks where RT-PCR was used. In a hospital for older people in Japan, Honda et al. found that all eight inpatients $(100 \%)$ in the same day-care room were RTI HMPV positive, while Tu et. al. found 10 of 13 patients (77\%) HMPV positive, in a psychiatric ward of an armed-forces general hospital in Taiwan $[12,15]$. All studies included relatively small numbers of patients ranging from eight to 18 patients. The differences in proportions might partly be explained by the different settings (residential care facilities for elderly versus hospital settings). On the other hand, in this outbreak investigation we 
sampled only staff and residents fulfilling the clinical case definition. Asymptomatic cases and less severe cases are therefore missed.

Follow-up respiratory samples showed that viral RNA remained detectable in residents during a relatively long period after disease onset, ranging from at least nine to 17 days. Whether this correlates with transmission of the virus is unknown. Because outbreak control measures were taken, the absence of new cases could not be used as a parameter for this. Alternatively, virus isolations by culture could be used as a surrogate parameter. However, isolation of HMPV by culture is relatively difficult due to its slow growth and mild cytopathic effects. Since viral culture remained negative in follow-up samples, it is possible that non-infectious viral particles/RNA fragments in cell debris from the lower lungs, could explain the positive rRT-PCR results in the follow-up samples.

We took infection control measures similar to those taken in case of RSV infection: clinical cases were cared for in isolation until clinical recovery and strict hand hygiene was applied. This approach seems justified given the probability of a relative long period of viral shedding. Also, after control measures were taken, new clinical cases only occurred in the following week, while they occurred during five weeks before the outbreak was notified. This is shorter than Boivin et al. reported [11]. In the later outbreak only dropletand contact precautions were taken and new cases occurred for at least two weeks.

When serological results were combined with rRTPCR results, four additional confirmed cases of HMPV infection and six probable cases were identified. The differences between rRT-PCR and serological results might be explained in various ways. Most likely, timing of sampling relative to onset of disease could explain these findings. Alternatively, sampling error or variations in the time of viral shedding might play some role. Possibly, more cases could have been confirmed as the sensitivity and specificity to detect IgM and IgG antibody titres with fixed and permeabilised infected cell monolayers is lower compared to enzyme-linked immunosorbent assay (ELISA), but HMPV ELISAs for both IgM and IgG were not in use as a diagnostic tool. Nevertheless, it is clear that a combined approach of serology and rRT-PCR has added diagnostic value.

For the early stage of an outbreak, serology by itself cannot be used as the interval between virus spreading

\section{TABLE 2}

Final case classification and laboratory results of clinical cases, outbreak of human metapneumovirus in a residential care facility for elderly in Utrecht, the Netherlands, January-March 2010 (n=23)

\begin{tabular}{|c|c|c|c|c|}
\hline Case & $\begin{array}{l}\text { Final case } \\
\text { classification }\end{array}$ & $\begin{array}{l}\text { HMPV rRT-PCR } \\
\text { Ct-value }\end{array}$ & $\begin{array}{l}\text { First serum sample } \\
\text { Reciprocal IgG antibody titre }\end{array}$ & $\begin{array}{l}\text { Second serum sample } \\
\text { Reciprocal IgG antibody titre }\end{array}$ \\
\hline 1 & Confirmed & $26^{a}$ & $\ll 16$ & 256 \\
\hline 2 & Confirmed & 33 & $<16(\operatorname{IgM} 256)$ & 1,024 \\
\hline 3 & Confirmed & 36 & $\langle 16$ & 1,024 \\
\hline 4 & Confirmed & 33 & $\ll 16$ & Deceased \\
\hline 5 & Confirmed & 38 & $<16$ & ND \\
\hline 6 & Confirmed & Negative & $\ll 16$ & 1,024 \\
\hline 7 & Confirmed & Negative & $\ll 16$ & 512 \\
\hline 8 & Confirmed & Negative & $\langle 16$ & 256 \\
\hline 9 & Confirmed & Negative & $\langle 16$ & 64 \\
\hline 10 & Probable & ND & $>1,024$ & ND \\
\hline 11 & Probable & ND & 21,024 & ND \\
\hline 12 & Probable & ND & $>1,024$ & ND \\
\hline 13 & Probable & ND & 256 & ND \\
\hline 14 & Probable & ND & 256 & ND \\
\hline 15 & Probable & Negative & 256 & ND \\
\hline 16 & Possible & Negative & 16 & ND \\
\hline 17 & Possible & Negative & Sample not provided & Sample not provided \\
\hline 18 & Possible & Sample not provided & Sample not provided & Sample not provided \\
\hline 19 & Possible & Deceased & Deceased & Deceased \\
\hline 20 & Possible & Deceased & Deceased & Deceased \\
\hline 21 & Possible & Deceased & Deceased & Deceased \\
\hline 22 & Non-case & ND & 64 & 16 \\
\hline 23 & Non-case & ND & 16 & 16 \\
\hline
\end{tabular}

ND: not determined; HMPV: Human metapneumovirus; rRT-PCR: real-time Reverse Transcriptase Polymerase Chain Reaction.

a Viral culture positive. 
and detection of HMPV-specific IgM and IgG antibodies is too long to manage the outbreak, especially considering immunosenescence in elderly [20]. Based on the present data, we would recommend a combined diagnostic approach, in which a direct IFA test is coupled to rRT-PCR. IFA is a rapid and useful test for the diagnosis of HMPV infections in outbreaks. However, the sensitivity of IFA is lower than that of rRT-PCR and needs to be validated before use [21]. While the results of the IFA, available within two hours, are validated by rRTPCR, appropriate measures can be taken. Furthermore, standardised timing of sample collection (time after onset of disease, time intervals between samples) for serology clearly contributes to the final identification of cases in outbreak studies.

This outbreak exemplifies that HMPV can cause severe disease. The majority of the resident cases were treated for pneumonia and some of them were admitted to the hospital. Several studies have reported mortality among elderly due to $\operatorname{HMPV}[3,11,15,22]$. Although none of the probable or confirmed HMPV resident cases in this outbreak died because of the HMPV infection, it is very likely that HMPV caused or contributed to the death of at least one of the three possible resident cases that were admitted to hospital and died there before the outbreak was notified. After all, the grand majority of the resident cases with laboratory results turned out to be probable or confirmed HMPV cases (13 of 14 clinical cases), making it highly probable that at least one of the three clinical cases, who

\section{FIGURE 4}

Flowchart of the laboratory investigations and results, outbreak of human metapneumovirus in a residential care facility for the elderly, Utrecht, the Netherlands, JanuaryMarch 2010

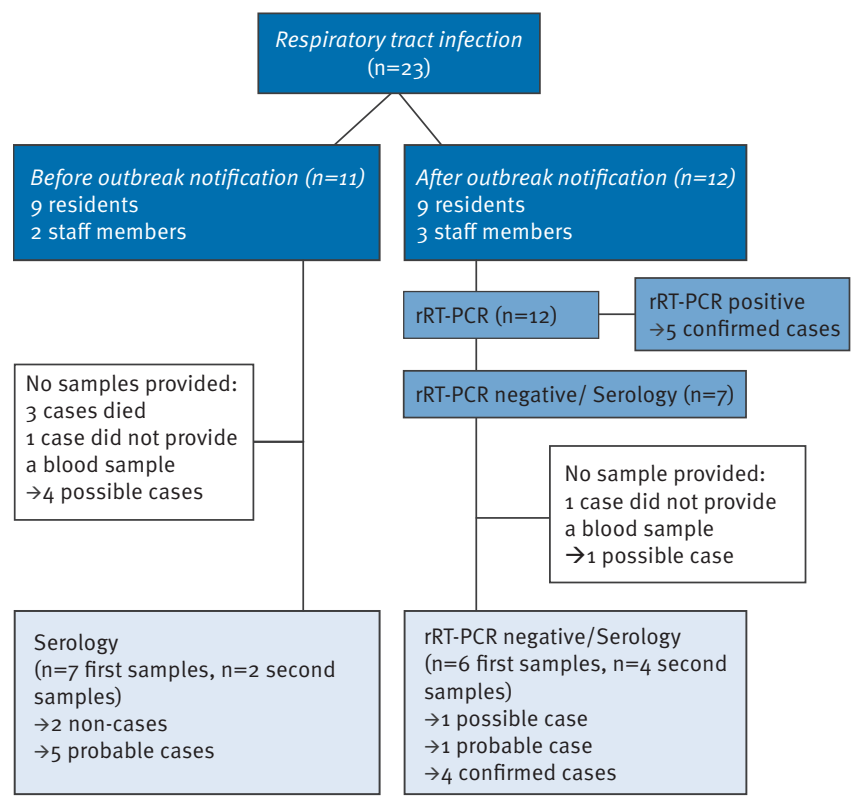

rRT-PCR: real-time Reverse Transcriptase Polymerase Chain Reaction. were admitted to hospital and died there before the outbreak was notified, had a HMPV infection too.

A further question is how the virus was spread. As both residents and staff members were affected, both groups could have transmitted the virus to others. Direct spread among residents is not very likely as they all have their own apartment, but it cannot be ruled out as they do share common facilities. Staff members, on one hand, could have easily transmitted the virus as they do work over all floors, but on the other hand they form a small minority under the (probable or confirmed) final cases. Another possibility is that affected staff members who did not fulfil the clinical case definition (especially the fever criterion) were involved in the transmission, since symptoms of HMPV infection are dependent on age and health of the host. It is therefore possible that staff members - (much) younger and healthier compared to residents - were infected with HMPV, but developed only minor symptoms and continued to work and in doing so, could have spread the virus. Whether this hypothesis holds, can only be studied in an outbreak in which not only persons fulfilling the clinical case definition are included, but (a sample of) those not fulfilling that definition as well.

In conclusion, this article describes an outbreak of HMPV in a residential care facility for elderly with an attack rate of $5-13 \%$ among residents, with severe disease and probable mortality. It is of interest that follow-up of rRT-PCR positive cases suggests a relatively long period of viral shedding. This should be considered when applying infection control measures.

\section{Acknowledgments}

We thank residents and staff members of the residential care facility - especially Angelique van der Burgh and Els van Schoot -, Georgina Aron and Rik de Swart from the Erasmus Medical Centre, Rotterdam, Emma Millar from Queen's University Belfast in Belfast, Northern Ireland and Sjon Oudejans from the Diakonessenhuis, Utrecht for their technical assistance and/or advices in this investigation.

\section{References}

1. van den Hoogen BG, de Jong JC, Groen J, Kuiken T, de Groot R, Fouchier RA, et al. A newly discovered human pneumovirus isolated from young children with respiratory tract disease. Nat Med. 2001;7(6):719-24.

2. van den Hoogen BG, Herfst S, Sprong L, Cane PA, Forleo-Neto E, de Swart RL, et al. Antigenic and genetic variability of human metapneumoviruses. Emerg Infect Dis. 2004;10(4):658-66.

3. van den Hoogen BG. Respiratory tract infection due to human metapneumovirus among elderly patients. Clin Infect Dis. 2007;44(9):1159-60.

4. Williams JV, Wang CK, Yang CF, Tollefson SJ, House FS, Heck JM, et al. The role of human metapneumovirus in upper respiratory tract infections in children: a 20 -year experience. J Infect Dis. 2006;193(3):387-95.

5. Aberle JH, Aberle SW, Redlberger-Fritz M, Sandhofer MJ, Popow-Kraupp T. Human metapneumovirus subgroup changes and seasonality during epidemics. Pediatr Infect Dis J. 2010;29(11):1016-8.

6. Peiris JS, Tang WH, Chan KH, Khong PL, Guan Y, Lau $\mathrm{YL}$, et al. Children with respiratory disease associated 
with metapneumovirus in Hong Kong. Emerg Infect Dis. 2003;9(6):628-33.

7. Boivin G, Abed Y, Pelletier G, Ruel L, Moisan D, Cote S, et al. Virological features and clinical manifestations associated with human metapneumovirus: a new paramyxovirus responsible for acute respiratory-tract infections in all age groups. J Infect Dis. 2002;186(9):1330-4.

8. Crowe JE, Jr. Human metapneumovirus as a major cause of human respiratory tract disease. Pediatr Infect Dis J. 2004;23(11 Suppl):S215-21.

9. Hamelin ME, Prince GA, Boivin G. Effect of ribavirin and glucocorticoid treatment in a mouse model of human metapneumovirus infection. Antimicrob Agents Chemother. 2006;50(2):774-7.

10. Wyde PR, Chetty SN, Jewell AM, Boivin G, Piedra PA. Comparison of the inhibition of human metapneumovirus and respiratory syncytial virus by ribavirin and immune serum globulin in vitro. Antiviral Res. 2003;60(1):51-9.

11. Boivin G, De Serres G, Hamelin ME, Cote S, Argouin M, Tremblay G, et al. An outbreak of severe respiratory tract infection due to human metapneumovirus in a long-term care facility. Clin Infect Dis. 2007;44(9):1152-8.

12. Honda H, Iwahashi J, Kashiwagi T, Imamura Y, Hamada $\mathrm{N}$, Anraku T, et al. Outbreak of human metapneumovirus infection in elderly inpatients in Japan. J Am Geriatr Soc. 2006;54(1):177-80.

13. Louie JK, Schnurr DP, Pan CY, Kiang D, Carter C, Tougaw S, et al. A summer outbreak of human metapneumovirus infection in a long-term-care facility. J Infect Dis. 2007;196(5):705-8.

14. Osbourn M, McPhie KA, Ratnamohan VM, Dwyer DE, Durrheim DN. Outbreak of human metapneumovirus infection in a residential aged care facility. Commun Dis Intell. 2009;33(1):38-40.

15. Tu CC, Chen LK, Lee YS, Ko CF, Chen CM, Yang HH, et al. An outbreak of human metapneumovirus infection in hospitalized psychiatric adult patients in Taiwan. Scand J Infect Dis. 2009;41(5):363-7.

16. Maertzdorf J, Wang CK, Brown JB, Quinto JD, Chu M, de Graaf $M$, et al. Real-time reverse transcriptase PCR assay for detection of human metapneumoviruses from all known genetic lineages. J Clin Microbiol. 2004;42(3):981-6.

17. Gunson RN, Collins TC, Carman WF. Real-time RT-PCR detection of 12 respiratory viral infections in four triplex reactions. J Clin Virol. 2005;33(4):341-4.

18. van de Pol AC, van Loon AM, Wolfs TF, Jansen NJ, Nijhuis $M$, Breteler EK, et al. Increased detection of respiratory syncytial virus, influenza viruses, parainfluenza viruses, and adenoviruses with real-time PCR in samples from patients with respiratory symptoms. J Clin Microbiol. 2007;45(7):2260-2.

19. Schutten M, Pas S. Protocol voor Influenzavirus real-time RT-PCR ErasmusMC. Wijzigingsdatum: 24-02-2012 [Protocol for influenza virus real-time RT-PCR ErasmusMC. Revision on: 24-02-2012]. Rotterdam: Erasmus Medisch Centrum; $21 \mathrm{Apr}$ 2011. Dutch. Available from: http://www.rivm.nl/Bibliotheek/ Professioneel Praktisch/Protocollen/Infectieziekten/ Protocol_voor_Influenzavirus_real_time_RT_PCR_ErasmusMC

20. Larbi A, Franceschi C, Mazzatti D, Solana R, Wikby A, Pawelec G. Aging of the immune system as a prognostic factor for human longevity. Physiology (Bethesda). 2008;23:64-74.

21. Ebihara T, Endo R, Ma X, Ishiguro N, Kikuta H. Detection of human metapneumovirus antigens in nasopharyngeal secretions by an immunofluorescent-antibody test. J Clin Microbiol. 2005;43(3):1138-41.

22. O’Gorman C, McHenry E, Coyle PV. Human metapneumovirus in adults: a short case series. Eur J Clin Microbiol Infect Dis. 2006;25(3):190-2. 